\title{
Curvature-Corrected Hardness Measurement Technique for Nanoindentation of Bonding Wire
}

\author{
Shugo Miyake, ${ }^{* \dagger}$ Emi Sasaki, Takaaki Kato, and Takahiro Namazu ${ }^{1}$ \\ Kobelco Research Institute, Inc., 1-5-5 Takatsukadai, Nishi-ku, Kobe, Hyogo 651-2271, Japan \\ ${ }^{1}$ Department of Mechanical Engineering, University of Hyogo, 2167 Shosha, Himeji, Hyogo 671-2201, Japan
}

(Received September 17, 2015; accepted November 12, 2015)

Keywords: bonding wire, hardness test, nanoindentation, curvature correction, contact area

In this article, we report a geometric study of the contact area for the hardness test of gold bonding wires using nanoindentation testing equipment based on the Oliver and Pharr method (OP method), which is frequently used to measure the hardness and elastic modulus. Since the OP method assumes that the sample surface is flat, the contact area detected by the OP method is underestimated, especially for a curved surface. We developed a contact area correcting method for nanoindentation testing, considering the geometric configuration during tip indentation on a curved surface, by theoretical analysis and experiment using nanoindentation testing equipment. Analysis results show that the area ratio (real contact area/contact area detected by the OP method) significantly decreases with decreasing wire diameter and increasing displacement into the surface, and we found the offset values to correct the contact area detected with the OP method for different wire diameters. Furthermore, nanoindentation testing was carried out on commercially available gold bonding wires with diameters of 30 and $50 \mu \mathrm{m}$. Measurement results show that the slopes of hardness-displacement curves slightly decrease with increasing displacement into the surface owing to the underestimated contact area. Then, we applied the analyzed offset value to the measured contact area to correct the obtained hardness. The corrected hardnesses of 30- and 50- $\mu \mathrm{m}$-diameter wires are approximately 0.8 and $0.9 \mathrm{GPa}$, respectively. These corrected values show an increase of $25-30 \%$ compared with the uncorrected values.

\section{Introduction}

To improve various electronic devices and components such as transistors, diodes, resistors and integrated circuits, their materials and wire bonding technology are applied with an improved electronic packaging design. In particular, mechanical properties of bonding wires should be improved to obtain higher performance characteristics such as higher reliability, strength, elongation and cost reduction with smaller wire diameters. ${ }^{(1-4)}$ We know from our experience that hardness is approximately three times as large as strength. Hence, the hardness test is widely adopted and used as a simplified practical mechanical testing method for determining the strength properties of various materials. In particular, the nanoindentation method has attracted the attention of engineers in the development of new small-scale electronic devices. Recently, the nanoindentation method combined with the Oliver and Pharr method (OP method) using a Berkovich indenter tip has

${ }^{*}$ Corresponding author: e-mail: miyake@kobe-kosen.ac.jp

Current address: Department of Mechanical Engineering, Kobe City College of Technology 
become the most popular technique..$^{(5-13)}$ The OP method was developed in 1992 for measurements of the hardness and elastic modulus of isotropic metals and amorphous materials such as aluminum, tungsten, soda-lime glass, fused silica, quartz and sapphire. ${ }^{(5)}$ One of its advantages is that hardness can be measured without the observation of the indented impression, but can directly be determined from the indentation load and displacement. ${ }^{(14-17)}$ With high-resolution testing equipment that involves micro-newton load sensitivity and nanometer displacement sensitivity, the method can be applied to measure the hardnesses of microscale thin films, fine structure materials and shallow regions. ${ }^{(18-27)}$ Since the sample surface is assumed to be flat in the method, the determined displacement of the indenter tip can be derived from the corresponding projected area of the contact area. However, the sample surface such as a bonding wire is not always exactly flat against indenter tips. ${ }^{(28-31)}$ Therefore, it is possible that the hardness measured by the nanoindentation method with the OP method might result in the underestimation of the hardness of the bonding wire. For this reason, it is essential to determine the real contact area correctly. There are a number of reports on curved-surface samples. ${ }^{(32-35)}$ However, these reports were not targeted at bonding wires.

In this paper, an approach to correcting the real contact area considering a curved surface based on the nanoindentation method with the OP method for bonding wires is presented by considering the geometric configuration of the contact between the indenter tip and the curved surface of the bonding wire. The ratio of the real contact area of the bonding wire to the ideal contact area, which is assumed to correspond to the indentation of the flat surface, is analyzed to correct measured values. Then, the experimental results of curvature-corrected hardness measurement are shown in comparison with the results obtained by the OP method for commercially available gold bonding wires.

\section{Correction Method}

A schematic of the indentation process from the viewpoint of the cross section of the indented impression is shown in Fig. 1. It is assumed that a Berkovich triangular pyramidal indenter tip forms an indent in the flat surface, which is an isotropic bulk elastic-plastic material. The depth $h_{\mathrm{c}}$ formed by contact between the indenter tip and the surface is given by

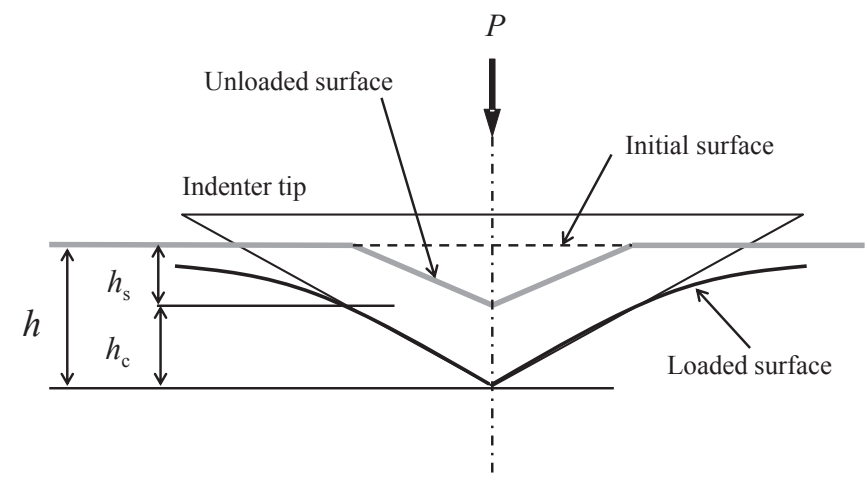

Fig. 1. Schematic of the indentation process from the viewpoint of the cross section and parameters. 


$$
h_{\mathrm{c}}=h-h_{\mathrm{s}},
$$

where $h$ is the amount of displacement from the initial undeformed surface and $h_{\mathrm{s}}$ is the amount of sink-in. Here, the amount of sink-in, $h_{\mathrm{s}}$, is given by

$$
h_{\mathrm{s}}=\varepsilon \frac{P_{\max }}{S},
$$

where $\varepsilon$ is a constant that depends on the shape of the indenter tip. ${ }^{(5-8,31,36,37)} P_{\max }$ is the maximum load and $S$ is the elastic unloading stiffness. Figure 2 shows a schematic of the representative indentation load-displacement curve $(P-h$ curve). $S$ is defined as the initial slope $(d P / d h)$ of the unloading curve.

The contact area $A$ corresponds to the projected area of the indentation impression and is related to $h_{\mathrm{c}}$ as

$$
A=F\left(h_{\mathrm{c}}\right)=24.56 h_{\mathrm{c}}^{2}
$$

and the hardness $H$ is also defined by

$$
H=\frac{P_{\max }}{A} .
$$

In practical use, the hardness is derived automatically by the analysis of a typical nanoindentation system regardless of the curvature of the sample surface.

Here, we discuss an aspect of contact between the Berkovich indentation tip and the curved surface. Figure 3 shows a schematic of the projected contact geometry for a thin wire indented by a Berkovich indentation tip from the viewpoint of the cross section of the wire. It is assumed that one of the apexes of the indentation impression corresponds to the ridge line of the curved surface.

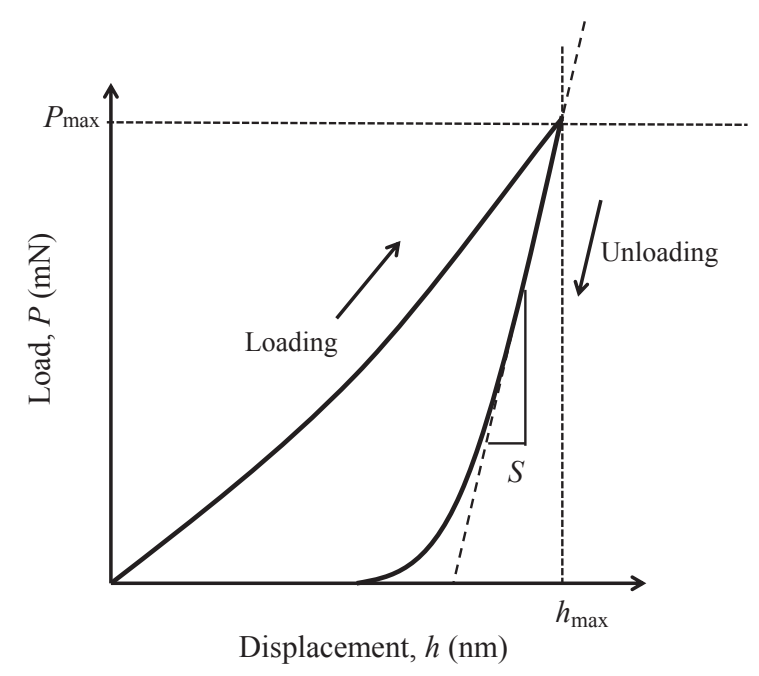

Fig. 2. Schematic of indentation load-displacement curve. 


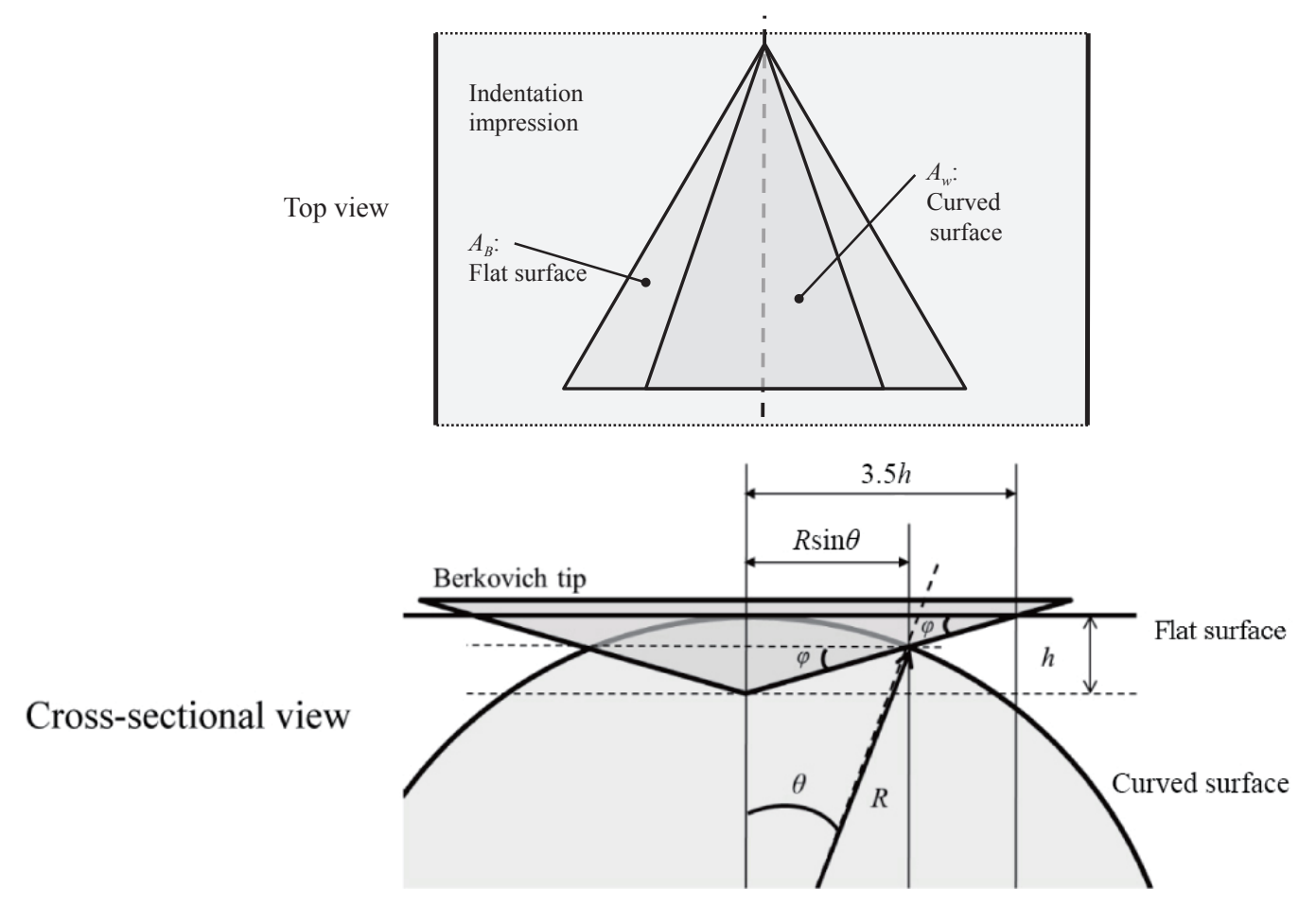

Fig. 3. Schematic of projected contact geometry for thin wire indented by a Berkovich tip.

In the case of this alignment, indentation impressions of flat and curved surfaces are shown in the bird's-eye view in the inset of Fig. 3. For the flat surface, the indentation impression is an equilateral triangle. On the other hand, it is an isosceles triangle for the curved surface. Therefore, it is possible that the difference in the projected contact area of the indentation impressions between the flat and curved surfaces is proportional to the side length of the triangle on the circumference of the wire as an approximate solution.

For the numerical analysis of the real contact area between the indentation tip and the curved surface, these surfaces must be modeled as mathematical functions. However, this analysis procedure is very complicated.

Here, as mentioned above, we suggest a simplification to obtain the solution. The ratio of the projected contact area of the curved surface to that of the flat surface is given as

$$
A_{\mathrm{W}}: A_{\mathrm{B}}=R \sin \theta: 7 h / 2,
$$

where $A_{\mathrm{B}}$ is the projected area of the flat surface, $A_{\mathrm{W}}$ is the projected area of the curved surface, $R$ is the wire radius and $\theta$ is half of the center angle of the intersecting points between the curved and tip surfaces in Fig. 3. In addition, it is possible that $h$ is the same as $h_{\mathrm{c}}$ and that $h_{\mathrm{s}}$ can be neglected. This is because the amount of sink-in on the curved surface is much smaller than that on the flat surface owing to the difference of the contact angle between the indenter tip and the sample surface. $R \sin \theta$ is given as 


$$
R \sin \theta=\frac{R(\cos \theta-1)+h}{\tan \varphi},
$$

where $\varphi$ is the angle of the flat surface relative to that of the tip surface. Hence, Eqs. (5) and (6) indicate that the calculated $R \sin \theta$ can provide a table of the area ratio $A_{\mathrm{W}} / A_{\mathrm{B}}$ for each $h$.

Figure 4 shows the dependence of the calculated area ratio on the displacement for 30 - and $50-\mu \mathrm{m}$-diameter bonding wires. Upon contact of the indentation tip with the sample surface, the area ratio is $1.0\left(A_{\mathrm{W}}=A_{\mathrm{B}}\right)$. This is because of the assumption that the surface is almost flat relative to the indentation tip when the radius of the edge of the indentation tip is much smaller than that of the bonding wire surface. The area ratio of the $30-\mu \mathrm{m}$-diameter bonding wire slightly decreases from 1.00 to 0.65 with increasing displacement. The area ratio of the $50-\mu \mathrm{m}$-diameter bonding wire also decreases from 1.00 to 0.73 . The curves indicate a nonlinear function, and we found a difference in the slope of the area ratio with the displacement between the shallow and deep indentations. It is likely that the slope of a deep indentation is more variable than that of a shallow indentation. Consequently, the relationship between the real and measured contact areas using the OP method is determined for the 30 - and $50-\mu \mathrm{m}$-diameter bonding wires.

\section{Hardness Measurement of Gold Bonding Wire}

Commercially available gold bonding wires with diameters of 30 and $50 \mu \mathrm{m}$ are prepared for hardness measurement by the nanoindentation method. Cross-sectioned images of these gold bonding wires observed by scanning electron microscopy (SEM) are shown in Fig. 5. In both the 30 - and $50-\mu \mathrm{m}$-diameter samples, crystal grains seem to be dispersed finely and isotropically at half of the outer diameter of the samples. On the other hand, at the inner center of the 10 - $\mu$ m-diameter sample, crystal grains are slightly larger than those at the outer center. However, this is a negligible effect when determining the hardness from the outer estimated area in this study. A 5-mm-long wire is rigidly fixed on a $10 \times 10 \times 1 \mathrm{~mm}^{3}$ glass substrate by epoxy adhesive as a specimen in the nanoindentation test. Here, it is assumed that there are no effects of the deformation of the epoxy

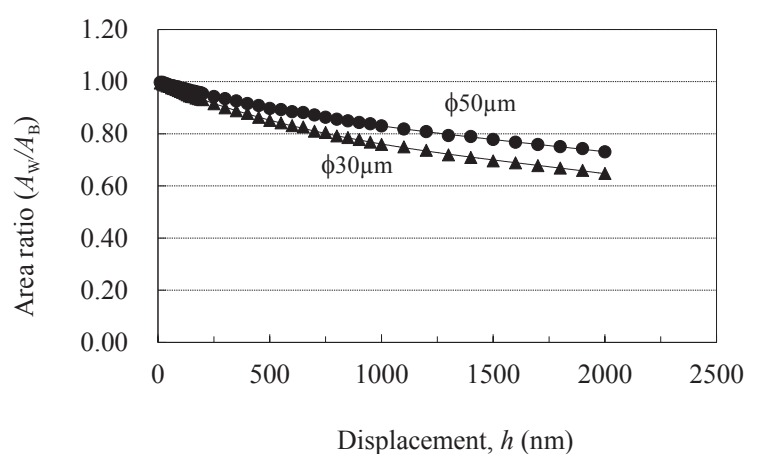

Fig. 4. Calculated results of the contact area ratio as a function of displacement for different wire diameters.

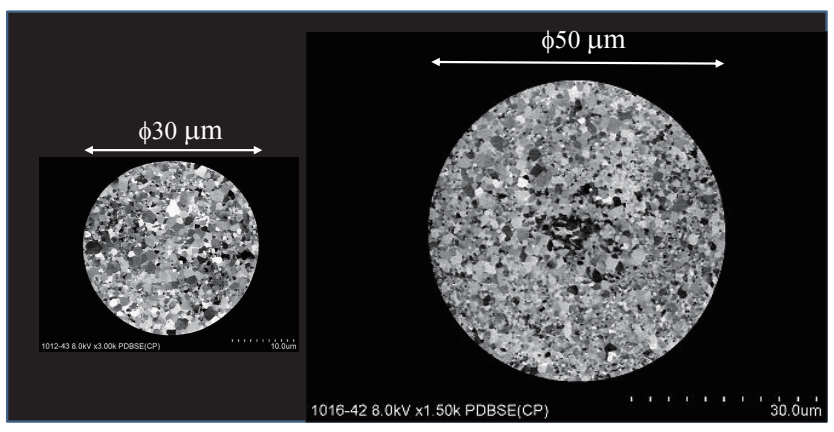

Fig. 5. Cross-sectional images of 30- and 50- $\mu \mathrm{m}-$ diameter binding wires observed by SEM. 
adhesive owing to the indentation load being much lower than the stiffness of the used epoxy adhesive. Half of the circumcolumnar surface of the fixed gold bonding wire is exposed to the atmosphere before the indentation of the tip as shown in Fig. 6(a). Moreover, a 100- $\mu$ m-diameter gold bonding wire is prepared and cut along a cross section to obtain a flat surface by mechanical polishing as a reference sample without a curved surface. The nanoindentation test is performed by the OP method using a G200 nanoindenter (MTS Systems Corporation) with a continuous stiffness measurement (CMS) instrument. The CSM instrument enables us to measure hardness as a function of depth due to the oscillating amplitude of the displacement with continuous loading onto the surface. The nanoindentation testing conditions are set as follows: strain rate of $0.05 / \mathrm{s}$, oscillating amplitude of displacement of $2 \mathrm{~nm}$, oscillating frequency of displacement of $45 \mathrm{~Hz}$ and maximum displacement of $2000 \mathrm{~nm}$. For the appropriate analysis of the contact area, the apex of the triangle of the indentation impression is aligned in the longitudinal direction of the wire in the test as shown in Fig. 6(b).

Figure 7 shows the measured hardness-displacement curves for gold bonding wires obtained by nanoindentation testing combined with the OP method. These results involve an average of 10 tests, and the error bars represent $1 \sigma$. The result of the $100-\mu$ m-diameter sample, which was measured at the flat surface, indicates that hardness increases with displacement at the early stage up to a displacement of $250 \mathrm{~nm}$. At a displacement of more than $250 \mathrm{~nm}$, the hardness is almost constant at approximately $0.75 \mathrm{GPa}$ regardless of the depth. On the other hand, the hardness changes with the displacement for the 30- and 50- $\mu \mathrm{m}$-diameter gold bonding wires, indicating different characteristics. For the 50 - $\mu$ m-diameter sample, hardness spikes from 0.7 to $0.9 \mathrm{GPa}$ at the early stage of indentation in the shallow region of displacement, and then it slightly decreases to 0.6 $\mathrm{GPa}$ at a displacement of $2000 \mathrm{~nm}$. The 30- $\mu \mathrm{m}$-diameter gold bonding wire also shows the same characteristics as the 50 - $\mu \mathrm{m}$-diameter gold bonding wire, but its hardness is $0.1 \mathrm{GPa}$ lower than that of the $50-\mu \mathrm{m}$-diameter sample in the entire displacement range in the test.

Figure 8 shows SEM images of the indentation impression of wires indented to a displacement of $2000 \mathrm{~nm}$ from the surface by a Berkovich indenter tip. An image of the flat surface of the $100-\mu \mathrm{m}$-diameter gold bonding wire is shown in Fig. 8(a). A pyramidal shape with the rectilinear

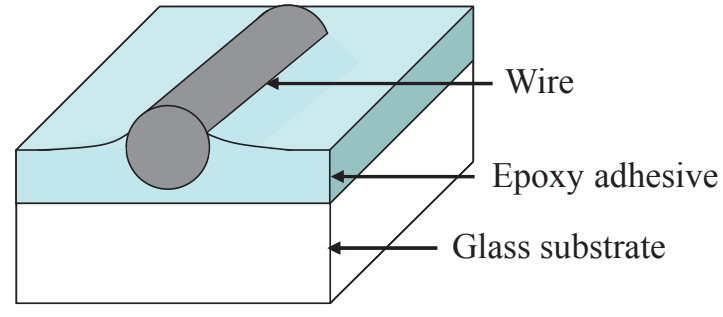

(a)

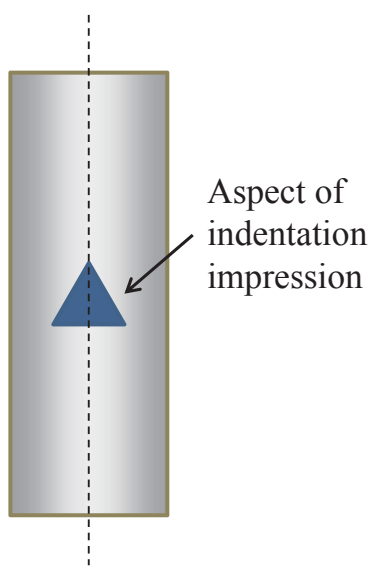

(b)

Fig. 6. (Color online) Schematics of tested specimen: (a) fixed wire and (b) aspect of indentation impression in longitudinal direction of the wire. 


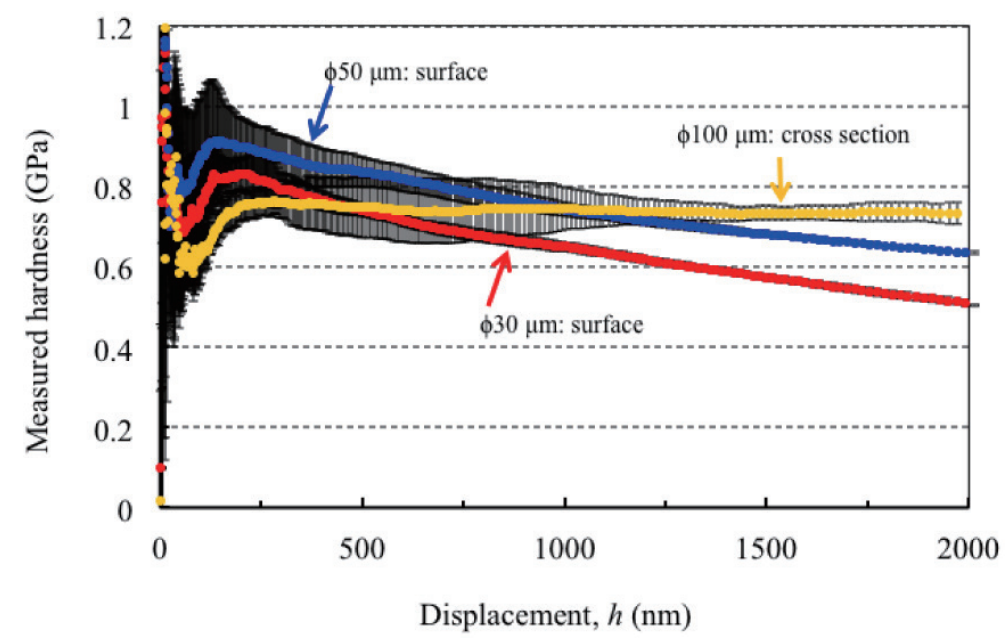

Fig. 7. (Color online) Hardness distribution in depth direction as a function of wire diameter measured by typical nanoindentation method.

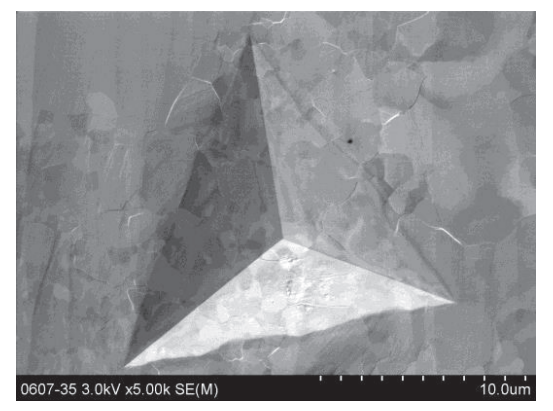

(a)

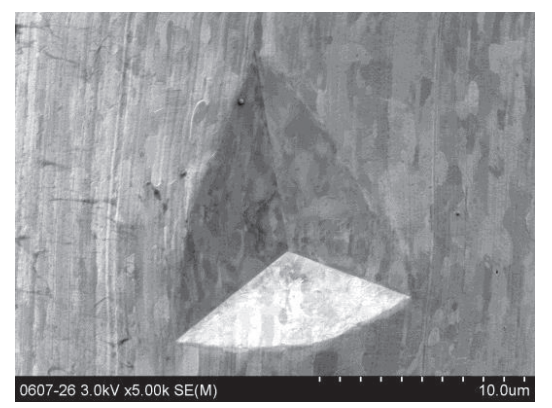

(b)

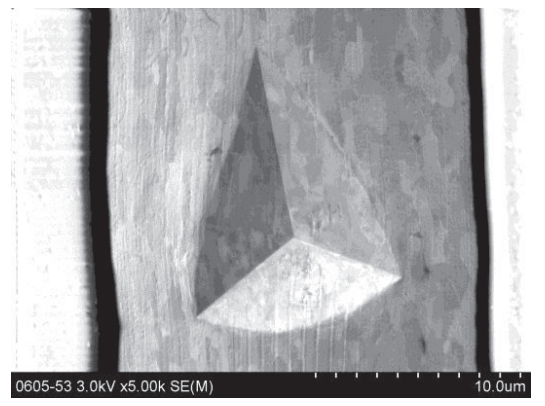

(c)

Fig. 8. Indentation impressions observed by SEM: (a) cross section of $100 \mu \mathrm{m}$ wire, (b) curved surface of $50 \mu \mathrm{m}$ wire, and (c) curved surface of $30 \mu \mathrm{m}$ wire.

side of the triangle is clearly observed, and this theoretically indicates an almost ideal indentation impression for the OP method. In Figs. 8(b) and 8(c), impressions indented on the curved surfaces of the 50- and 30- $\mu$-diameter wires are shown, respectively. Oval impressions with three remaining apexes are found in both images. It is found that the width of the oval indentation impression of the $30-\mu \mathrm{m}$-diameter wire is smaller than that of the $50-\mu \mathrm{m}$-diameter wire, and the indentations have the appearance of an isosceles triangle with a short side.

Figure 9 shows the measured and curvature-corrected hardnesses of the bonding wires. The corrected hardnesses of the 30- and 50- $\mu$ m-diameter gold bonding wires are approximately 0.8 and $0.9 \mathrm{GPa}$, respectively. These corrected values are 0.15 to $0.2 \mathrm{GPa}$ larger than the measured hardnesses, and the increase for the 30- $\mu$ m-diameter gold bonding wire is larger than that for the $50-\mu \mathrm{m}$-diameter gold bonding wire. The dependence of the displacement on hardness for each diameter slightly decreases with increasing displacement. However, this is a relaxed response to the noncorrected hardness distribution in the depth direction. Thus, the corrected hardness is close to the true value of the bonding wire with a small curvature. In future studies, this technique must be further improved for its use as a conventional method with high precision. 


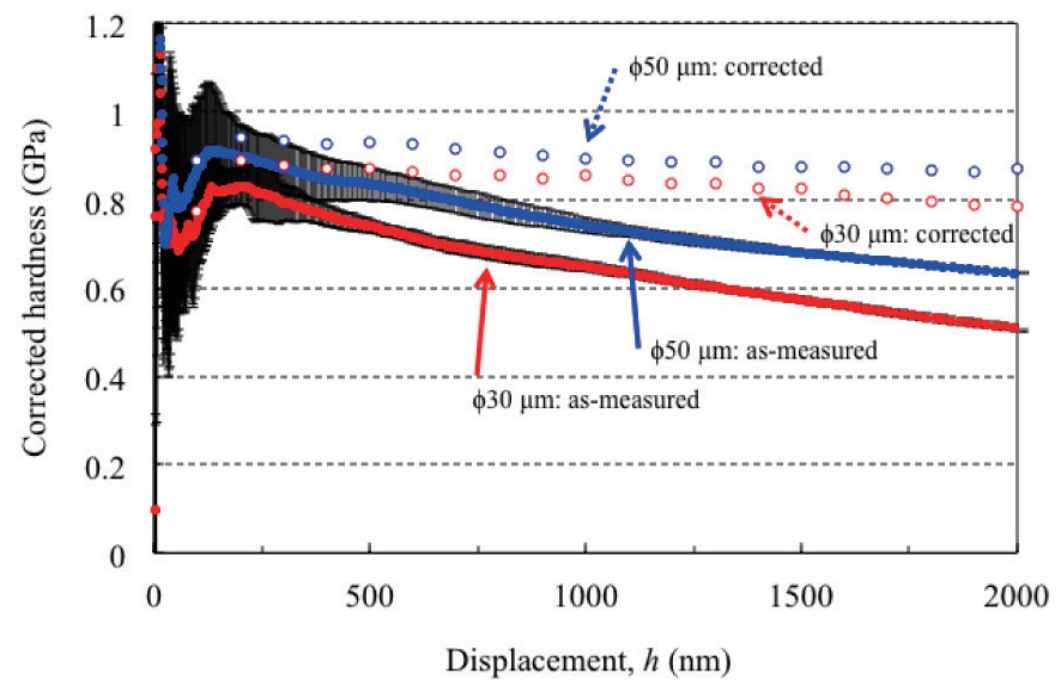

Fig. 9. (Color online) Hardness distribution in depth direction as a function of wire diameter estimated by curvature-corrected hardness measurement.

\section{Conclusions}

In this study, we performed curvature-corrected hardness measurement by the geometric analysis of gold bonding wires based on a nanoindentation method. We found that the area ratio $A_{\mathrm{W}} / A_{\mathrm{B}}$, which depends on the displacement into the surface, decreases with decreasing wire radius. For the hardness measurements of 30- and 50- $\mu \mathrm{m}$-diameter commercially available gold bonding wires, nanoindentation testing was carried out using the OP method, taking into account the corrected values derived by numerical calculation. Results show that the corrected values are $0.8 \mathrm{GPa}$ for the $30-\mu \mathrm{m}$-diameter wire and $0.9 \mathrm{GPa}$ for the $50-\mu \mathrm{m}$-diameter wire, which are 0.15 to $0.2 \mathrm{GPa}$ larger than the noncorrected values. The curvature correction technique for nanoindentation is expected to be useful for hardness estimations.

\section{References}

1 M. Shah, Z. Kaiyang, and A. A. Tay: J. Electron. Packag. 126 (2004) 87.

2 X. Fan, K. Qian, T. Wang, Y. Cong, M. Zhao, B. Zhang, and J. Wang: Proceedings of 2009 IEEE Electronic Packaging Technology \& High Density Packaging (IEEE, 2009) p. 790.

3 M. N. Zulkifli, S. Abdullah, N. K. Othman, and A. Jalar: Gold Bull. 45 (2012) 115.

4 A. Jalar, M. N. Zulkifli, and S. Abdullah: Adv. Mater. Res. 148 (2011) 1163.

5 W. C. Oliver and G. M. Pharr: J. Mater. Res. 7 (1992) 1564.

6 T. C. Chou, T. G. Nieh, T. Y. Tsui, G. M. Pharr, and W. C. Oliver: J. Mater. Res. 7 (1992) 2765.

7 T. C. Chou, T. G. Nieh, S. D. McAdams, G. M. Pharr, and W. C. Oliver: J. Mater. Res. 7 (1992) 2774.

8 G. M. Pharr and W. C. Oliver: MRS Bull. 17 (1992) 28.

9 T. Y. Tsui, W. C. Oliver, and G. M. Pharr: J. Mater. Res. 11 (1996) 752.

10 A. Bolshakov, W. C. Oliver, and G. M. Pharr: J. Mater. Res. 11 (1996) 760.

11 T. Y. Tsui, G. M. Pharr, W. C. Oliver, C. S. Bhatia, R. L. White, S. Anders, A. Anders, and I. G. Brown: MRS Proc. 383 (1995) p. 447. 
12 T. F. Page, W. C. Oliver, and C. J. McHargue: J. Mater. Res. 7 (1992) 450.

13 A. Bolshakov, W. C. Oliver, and G. M. Pharr: MRS Proc. 356 (1994) p. 675.

14 G. M. Pharr and A. Bolshakov: J. Mater. Res. 17 (2002) 2660.

15 S. V. Hainsworth, H. W. Chandler, and T. F. Page: J. Mater. Res. 11 (1996) 1987.

16 J. C. Hay, A. Bolshakov, and G. M. Pharr: J. Mater. Res. 14 (1999) 2296.

17 M. Jürgen: J. Mater. Res. 17 (2002) 502.

18 S. Ranjana and D. N. William: Acta Mater. 50 (2002) 23.

19 J. M. Sanchez, S. El-Mansy, B. Sun, T. Scherban, N. Fang, D. Pantuso, W. Ford, M. R. Elizalde, J. M. Martinez-Esnaola, A. Martine-Meizoso, J. Gil-Sevillano, M Fuentes, and J. Maiz: Acta Mater. 47 (1999) 4405.

20 K. Koiwa, N. Shishido, C. Chen, M. Omiya, S. Kamiya, H. Sato, M. Nishida, T. Suzuki, T. Nakamura, T. Suzuki, and T. Nokuo: Scr. Mater. 111 (2015) 94.

21 R. Ge, X. Hou, K. Brookshire, N. R. Krishnan, D. Silva, J. Bumgarner, L. Yinong, L. Faraone, and M. Martyniuk: Optoelectronic and Microelectronic Materials \& Devices (COMMAD), 2014 Conference (IEEE, 2014) p. 210.

22 A. Dey and A. K. Mukhopadhyay: Nanoindentation Study on Hard Ceramic Thin Films: TiN: Nanoindentation of Brittle Solids (CRC Press, 2014) p. 293.

23 J. Dong, H. Jiang, L. Sun, and Z. J. Long: Surf. Coat. Technol. 261 (2015) 208.

24 D. Maharaj and B. Bharat: Beilstein J. Nanotechnol. 5 (2014) 822.

25 S.-R. Jian and Y.-Y. Lin: J. Alloys Compd. 590 (2014) 153.

26 Y. Wei, W. Xuezheng, and Z. Manhong: J. Mater. Res. 19 (2004) 208.

27 Q. Ma and D. R. Clarke: J. Mater. Res. 10 (1995) 853.

28 M. Miller, C. Bobko, M. Vandamme, and F. J. Ulm: Cem. Concr. Res. 38 (2008) 467.

29 M. S. Bobji and S. K. Biswas: J. Mater. Res. 14 (1999) 2259.

30 M. S. Bobji and S. K. Biswas:.J. Mater. Res. 13 (1998) 3227.

31 K. W. McElhaney, J. J. Vlassak, and W. D. Nix: J. Mater. Res. 13 (1998) 1300.

32 Q. P. McAllister and J. W. Gillespie, Jr: J. Mater. Res. 27 (2012) 197.

33 G. Bingzheng: Int. Mech. Sci. 40 (1998) 1191.

34 N. Lonnroth, C. L. Muhlstein, C. Pantano, and Y. Yue: J. Non-Cryst. Solids 354 (2008) 3887.

35 D. P. Cole and K. E. Strawhecker: J. Mater. Res. 29 (2014) 1104.

36 S. Q. Shu, Y. Yang, T. Fu, C. S. Wen, and J. Lu: J. Mater. Res. 24 (2009) 1054.

37 H. Bei, E. P. George, J. L. Hay, and G. M. Pharr: Phys. Rev. Lett. 95 (2005) 045501. 